\title{
Outcome of peripheral iridotomy in subjects with uveitis
}

\author{
Thomas David Betts (1) , ${ }^{1,2}$ Joanne L Sims, ${ }^{2}$ Sonya L Bennett, ${ }^{2}$ Rachael L Niederer ${ }^{2}$
}

${ }^{1}$ Ophthalmology, Christchurch Hospital, Christchurch, New Zealand

2Ophthalmology, Greenlane Clinical Centre, Auckland, New Zealand

\section{Correspondence to} Dr Rachael L Niederer, Ophthalmology, Greenlane Clinical Centre, Auckland 1051, New Zealand; dr_rachnz@ yahoo.co.nz

Received 7 March 2019 Revised 29 May 2019

Accepted 27 June 2019 Published Online First 9 July 2019

\section{SLinked}

- http://dx.doi.org/10.1136/ bjophthalmol-2019-315221

Check for updates

(C) Author(s) (or their employer(s)) 2020. No commercial re-use. See rights and permissions. Published by BMJ.

To cite: Betts TD, Sims JL,

Bennett SL, et al.

$\mathrm{Br} J$ Ophthalmol

2020;104:8-10.

\section{ABSTRACT}

Background/aims Peripheral iridotomy (PI) may be required in subjects with uveitis to manage iris bombe, seclusio pupillae and primary angle closure glaucoma. The aim of this study was to identify risk factors for failure of both laser and surgical PIs in patients with uveitis and determine survival durations.

Methods Retrospective study of subjects with a history of uveitis undergoing yttrium-aluminium-garnet (YAG) laser or surgical PI at Auckland District Health Board over an 11-year period. Failure of PI was defined as loss of patency or recurrence of iris bombe. A mixed effects shared frailty model was constructed with PI nested within eyes nested within patients, to examine time to failure.

Results 131 Pls were performed in 52 eyes of 39 subjects during the study period (111 YAG PIs and 20 surgical PIs). Median age at time of PI was 46.6 years and $60.5 \%$ of subjects were female. HLAB27 positive uveitis was the most common diagnosis $(25.6 \%$ of subjects). Median survival time was 70 days for YAG PI and 11.0 years for surgical PI. On multivariate analysis, younger age at time of $\mathrm{PI}(\mathrm{HR} 0.933, \mathrm{p}<0.001)$ and iris bombe (HR 2.180, $p=0.046$ ) were associated with risk of failure. Surgical PI was associated with a lower risk of failure (HR 0.151, $p<0.001)$ compared with YAG PI. Glaucoma developed in 19 eyes (36.5\%), of which 13 required glaucoma surgery.

Conclusion Surgical PI had longer survival than YAG PI, and should be considered in subjects presenting with iris bombe and in young subjects with uveitis.

\section{INTRODUCTION}

Uveitis is a common inflammatory ocular disease and is the third leading cause of preventable blindness worldwide. ${ }^{1}$ Uveitis can be complicated by glaucoma, which may follow an aggressive course, with higher intraocular pressures (IOP) and more rapid progression of glaucomatous optic neuropathy $(\mathrm{GON}) .^{2}$ The mechanism of uveitic glaucoma (UG) is often mixed with open angle, closed angle and steroid-induced processes coexisting. ${ }^{3}$ The formation of synechial adhesion between the uveitic iris and lens can result in seclusio pupillae and iris bombe. This obstruction to normal intraocular aqueous flow induces a secondary angle closure with elevated IOP and potential peripheral anterior synechiae formation and GON.

The peripheral iridotomy (PI) has an established role in the prevention and treatment of this secondary angle-closure mechanism. Iridotomies are commonly performed using neodymium:yttrium-aluminiumgarnet (Nd:YAG) (YAG) laser or surgically. The limitation of this intervention has been previously reported, chiefly the high early failure rate. ${ }^{4}$ There is however paucity of information on the risk factors for failure of the iridotomy in uveitis.

This study was conducted to identify risk factors for failure of both laser and surgical PIs in patients with uveitis and determine survival durations.

\section{METHODS}

\section{Subject selection}

Notes were reviewed from a registry of 2366 subjects with uveitis seen in uveitis clinic (JLS and RLN) at Auckland District Health Board between 1 January 2008 and 31 December 2018 (ethics NTX/12/ EXP/085). All subjects with PI were eligible for inclusion. Subjects were excluded if the PI was performed prior to the onset of uveitis, or if there was no history of uveitis (eg, fellow eye). Unsuccessfully performed PIs (4/131) were excluded from survival analysis.

\section{Data collection}

Data were recorded onto a standardised pro forma, which captured subject demographics, presentation, indication for PI, type of procedure (YAG or surgical), the presence or not of inflammation at the time of the PI, complications, inflammation at the 1-month follow-up visit, time to failure or last follow-up and development of glaucoma, glaucoma surgery or loss of vision secondary to glaucoma. Glaucoma progression was determined from clinical examination documentation of optic nerve cup-disc ratio change and automated visual field deterioration.

Failure was defined as recurrence of iris bombe or loss of patency. We did not define failure as raised IOP, as some subjects developed UG in the presence of a patent PI. Subjects receiving more than one iridotomy in a single sitting had these included as separate events as the PIs potentially fail at different times. If a patient represented with iris bombe it was assumed all had failed. The statistical analysis allowed for clustering of the PIs.

\section{Statistical analysis}

Data were entered into an Excel spreadsheet and analysis was performed in STATA V.15 (StataCorp 2017, College Station, TX). Continuous data are reported as median (IQR and categorical data as number (\%)). To allow for concordance between eyes and also between repeat PIs performed on the same eye, we constructed a three-level mixed effects shared frailty survival model with PI nested within eyes nested within patients, to examine time to failure. The conditional HR is reported, which is the HR dependent on intereye and interpatient effects. A p value $\leq 0.05$ was considered significant. 


\begin{tabular}{ll}
\hline Table 1 & Subject demographics \\
\hline & $\mathrm{n}=39$ subjects \\
\hline Female & $23(60.5 \%)$ \\
\hline Age at diagnosis of uveitis & Median 42.6 (IQR 31.2-59.1) \\
\hline Age at first iridotomy & Median 46.6 (IQR 35.9-61.2) \\
\hline Ethnicity & \\
\hline Caucasian & $14(35.9 \%)$ \\
\hline Indian & $5(12.8 \%)$ \\
\hline Other Asian & $7(17.9 \%)$ \\
\hline Maori & $6(15.4 \%)$ \\
\hline Pacific Island Peoples & $6(15.4 \%)$ \\
\hline Middle Eastern & $1(2.6 \%)$ \\
\hline Anatomical classification of uveitis & \\
\hline Acute anterior uveitis & $13(33.3 \%)$ \\
\hline Chronic anterior uveitis & $11(28.2 \%)$ \\
\hline Chronic anterior and intermediate uveitis & $3(7.7 \%)$ \\
\hline Intermediate uveitis & $1(2.6 \%)$ \\
\hline Panuveitis & $11(28.2 \%)$ \\
\hline Diagnosis & \\
\hline Idiopathic & $9(23.1 \%)$ \\
\hline HLAB27 uveitis & $10(25.6 \%)$ \\
\hline Psoriatic arthritis & $2(5.1 \%)$ \\
\hline Viral anterior uveitis & $4(10.3 \%)$ \\
\hline Sarcoidosis & $4(10.3 \%)$ \\
\hline Tuberculosis & $5(12.8 \%)$ \\
\hline Vogt-Koyanagi-Harada disease & $3(7.7 \%)$ \\
\hline Uveitis-glaucoma-hyphaema syndrome & $1(2.6 \%)$ \\
\hline Syphilis & $1(2.6 \%)$ \\
\hline & \\
\hline
\end{tabular}

\section{RESULTS}

One hundred and thirty-one PIs in 52 eyes of 39 subjects were included for analysis with a total of 486.3 eye-years of follow-up after PI. Subject demographics are reported in table 1. One hundred and eleven YAG PIs and 20 surgical PIs were performed. Four surgical PIs were performed as the primary procedure (20.0\%), and in the other 16 they were performed following failure of YAG PI. Iris bombe $(n=91,69.5 \%)$ was the most common reason for PI, followed by primary angle closure/narrow angles $(n=23,17.6 \%)$, seclusio pupillae without bombe $(\mathrm{n}=15,11.5 \%)$, tube touch $(\mathrm{n}=1$, $0.8 \%)$ and iris chafe $(\mathrm{n}=1,0.8 \%)$. At the time of PI, there was active inflammation in 86 eyes (65.7\%).

Complications occurred following 31 (23.9\%) of PI and included increased inflammation in 14, hyphaema in 15 and

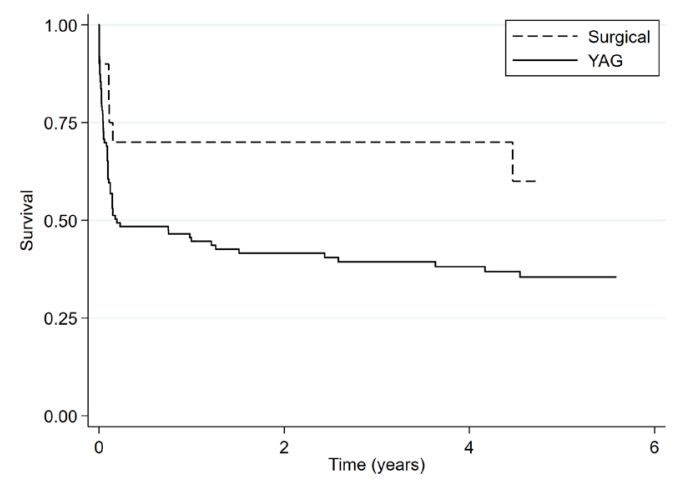

Figure 1 Kaplan-Meier survival graph comparing type of peripheral iridotomy. YAG, yttrium-aluminium-garnet laser.
Table 2 Risk factors for peripheral iridotomy failure

\begin{tabular}{lllll}
\hline & \multicolumn{2}{l}{ Univariate } & \multicolumn{2}{l}{ Multivariate } \\
\hline Risk factor & HR & P value & HR & P value \\
Age at PI & 0.931 & $<0.001$ & 0.933 & $<0.001$ \\
Female & 0.368 & 0.082 & & \\
Caucasian & 0.527 & 0.330 & & \\
Surgical PI & 0.198 & $<0.001$ & 0.151 & $<0.001$ \\
Bombe & 3.582 & 0.001 & 2.180 & 0.046 \\
First PI (compared with subsequent) & 0.918 & 0.814 & & \\
Inflammation at PI & 1.567 & 0.159 & & \\
Oral steroid & 2.421 & 0.036 & 1.745 & 0.246 \\
Inflammation 1 m & 2.252 & 0.033 & 1.418 & 0.331 \\
\hline
\end{tabular}

HR is conditional hazard ratio (conditional on random effects). $\mathrm{Pl}$, peripheral iridotomy.

increased IOP in 2. One subject developed a retinal detachment 1 month following PI and one subject developed herpes simplex virus keratitis 1 month after PI. Complications occurred in $24.5 \%$ of YAG PI and $20.0 \%$ of surgical PI, and this difference was not statistically significant $(\mathrm{p}=0.661)$.

The median number of PI per eye was 1 (IQR 1-3, range 1-21). Fourteen eyes (11 subjects) had $>2$ PIs performed per eye, on different sessions. Ethnicity of these subjects was Pacific Island People four subjects (six eyes), Maori three subjects (four eyes), East Asian two subjects (two eyes), Indian one subject (one eye) and Caucasian one subject (one eye). Diagnosis was idiopathic chronic anterior uveitis three subjects (four eyes), VogtKoyanagi-Harada syndrome two subjects (three eyes), HLAB27 uveitis four subjects (five eyes), tuberculosis-associated chronic anterior uveitis one subject (one eye) and sarcoid panuveitis one subject (one eye). One subject with Vogt-Koyanagi-Harada syndrome was an outlier with 21 PIs in one eye (3 surgical and 18 YAG), all of which failed and he ultimately had $360^{\circ}$ of iridocorneal touch and perception of light vision. This subject had repeated non-attendance of clinic appointments and poor compliance with treatment, often presenting after hours in iris bombe.

Median survival time was 70 days for YAG PI and 11.0 years for surgical PI (figure 1). A mixed effects shared frailty model was constructed with PI nested within eyes nested within patients, to examine time to failure. Univariate and multivariate analysis results are reported in table 2. On univariate analysis, younger age at time of PI, YAG PI, iris bombe, oral steroid use and inflammation at 1-month follow-up were all associated with an increased risk of failure. On multivariate analysis, only younger age at time of PI, YAG PI and iris bombe were associated with risk of failure. A Kaplan-Meier survival plot demonstrating risk of failure by indication for PI is given in figure 2. The median survival time for a PI performed for iris bombe was 35 days for YAG PI and 11.0 years for a surgical PI. Conversely, median survival for YAG PI for primary angle closure was 16.3 years, only one surgical PI was performed in this cohort, which did not fail over the follow-up period. The conditional HR for PI survival by age was 0.933 per year $(\mathrm{p}<0.001)$, meaning that for every increase in age of 10 years, there was a reduction in risk of failure by $49.8 \%$.

Analysis was repeated excluding the single outlying case with 21 PIs in one eye. The results on multivariate analysis were essentially unchanged, although with the outlier excluded, bombe did not reach statistical significance as a predictor of failure $(p=0.072)$.

No patients had pre-existing GON. In some cases there were limited examinations of glaucoma parameters secondary to 


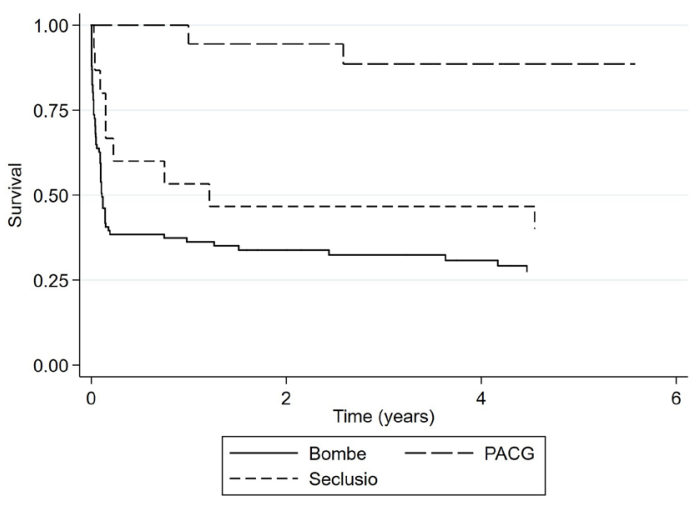

Figure 2 Kaplan-Meier survival graph comparing indication for peripheral iridotomy. PACG, primary angle closure glaucoma.

poor vision and opacity of optical media. During the follow-up period, 19 eyes (36.5\%) developed GON of which 13 (25.0\%) required glaucoma surgery. Glaucoma surgery was trabeculectomy with mitomycin $\mathrm{C}$ in nine eyes, Molteno tube in three eyes and Baerveldt tube in one eye. Seven eyes (13.5\%) developed decreased vision $\leq 6 / 15$ attributed to glaucoma, of which four had vision $\leq 6 / 60$. The subject with 21 failed PIs dropped his vision to perception of light, but there was no view of the disc and bilateral immobile pupils due to posterior synechiae, so the contributory role of glaucoma could not be ascertained.

\section{DISCUSSION}

In this retrospective study, 111 YAG PIs and 20 surgical PIs were performed. Sixteen (80.0\%) surgical PIs were performed as secondary procedures following the failure of initial YAG PI. The median survival time for a YAG PI in our study was 70 days and 11.0 years for a surgical PI. A quarter of patients had a complication following iridotomy formation, most frequently being an increase in inflammation or hyphaema.

In uveitis the products of intraocular inflammation in the aqueous stimulate the formation of posterior synechiae between the iris and lens capsule. With the formation of $360^{\circ}$ of synechiae adhesion the flow of aqueous into the anterior chamber is disturbed producing iris bombe and a secondary angle closure crisis.

Multivariate analysis indicates that iris bombe is associated with an increased risk of PI failure. Iris bombe is challenging to manage acutely and can result in irreversible anatomical and functional impairment. Aggressive control of inflammation in subjects at risk of iris bombe is important to prevent this complication, along with use of mydriatic agents to help break posterior synechiae when they form. In some instances, mydriatic agents alone may break an episode of iris bombe by resolving the seclusio pupillae. The use of tissue plasminogen activator intracamerally may also provide a useful adjunct in managing these difficult cases. ${ }^{5}$

It is of interest that we did not demonstrate an increased risk of failure with inflammation at time of PI (univariate analysis, $\mathrm{p}=0.159)$. A $50 \%$ increased rate of PI failure was shown on univariate analysis in those with active inflammation at the time of PI, but this did not reach statistical significance. Interestingly, it was inflammation in the month following the PI that was significant on univariate analysis $(p=0.033)$, reinforcing the value of tight inflammation control following PI.

In our series those patients who underwent a PI in the presence of iris bombe were managed most successfully with a surgical PI. The median survival for a YAG PI in the context of bombe was 35 days compared with 4.5 years for surgical PI. The authors recommend that those patients with iris bombe may undergo a YAG PI as a temporising measure but a surgical PI should be performed within a week as a definitive procedure.

Multivariate analysis found that those having a PI at a younger age were at increased risk of failure $(\mathrm{p}<0.001)$. This may be the result of more vigorous wound healing and inflammation in young patients, akin to poor survival of pre-mitomycin $\mathrm{C}$ trabeculectomy in younger patients. ${ }^{6}$ Given the demonstrated improved survival of surgical PI over YAG PI (11 years vs 70 days) it may be prudent to offer surgical iridotomy as a primary procedure in young patients and extra vigilance to postprocedure immunosuppression.

A further finding from this study was that subjects with a history of previous uveitis and concurrent narrow angles who underwent YAG PI did well with a median survival of 16.3 years. There are limited long-term survival data in patients with narrow angles without GON. Closure is reported to be rare and at 1 year in a prospective study of East Asian eye 98.1\% remained patent ${ }^{78}$

Despite careful follow-up and management of elevated IOP, $36.5 \%$ of eyes requiring PI developed GON, with a quarter of subjects requiring surgical intervention. Of the 19 eyes that developed GON, 7 (36.8\%) lost vision. All subjects with uveitis that require a PI should be comanaged between uveitis and glaucoma specialists to optimise control of inflammation and to ensure close monitoring of their angle and optic nerve.

This is the largest study to date conducted to identify risk factors for failure of both laser and surgical PIs in patients with uveitis. Young age, the use of ND:YAG laser and iris bombe were all statistically significant risk factors associated with increased risk of iridotomy failure. Overall survival was 70 days for YAG PI and 11.0 years for surgical PI. A significant proportion of eyes developed vision loss from glaucoma. We advocate early surgical PI in those with iris bombe and careful comanagement of these subjects with uveitis and glaucoma subspecialists.

Contributors Data collection and article composition by TDB. Statistical analysis by RLN. Draft reviews and research support throughout by JLS, SLB and RLN.

Funding This research received no specific grant from any funding agency in the public, commercial or not-for-profit sectors.

Competing interests None declared.

Patient consent for publication Not required.

Ethics approval Auckland District Health Board (ethics NTX/12/EXP/085).

Provenance and peer review Not commissioned; externally peer reviewed.

Data availability statement Data are available upon request.

ORCID iD

Thomas David Betts http://orcid.org/0000-0002-2791-7498

\section{REFERENCES}

1 Tsirouki T, Dastiridou A, Symeonidis C, et al. A focus on the epidemiology of uveitis. Ocul Immunol Inflamm 2018:26:2-16.

2 Siddique SS, Suelves AM, Baheti U, et al. Glaucoma and uveitis. Surv Ophthalmol 2013:58:1-10.

3 Muñoz-Negrete FJ, Moreno-Montañés J, Hernández-Martínez P, et al. Current approach in the diagnosis and management of uveitic glaucoma. Biomed Res Int 2015:2015:742792.

4 Spencer NA, Hall AJ, Stawell RJ. Nd:YAG laser iridotomy in uveitic glaucoma. Clin Exp Ophthalmol 2001;29:217-9.

5 Lerner LE, Patil AJ, Kenney MC, et al. Use of intraocular human recombinant tissue plasminogen activator as an adjunct treatment of posterior synechiae in patients with uveitis. Retin Cases Brief Rep 2012;6:290-3.

6 Skuta GL, Parrish RK. Wound healing in glaucoma filtering surgery. Surv Ophthalmol 1987;32:149-70.

7 Nolan WP, Foster PJ, Devereux JG, et al. YAG laser iridotomy treatment for primary angle closure in East Asian eyes. Br J Ophthalmol 2000;84:1255-9.

8 Quigley HA. Long-term follow-up of laser iridotomy. Ophthalmology 1981;88:218-24. 